From the Association of Departments of Family Medicine

\section{ADVANCING DIVERSITY, INCLUSION, AND HEALTH EQUITY TO THE NEXT LEVEL}

The Association of Departments of Family Medicine (ADFM), as part of its mission, has made diversity, inclusion, and health equity priorities. This mission is being carried out with adherence to the core values that are crucial to our member departments' teaching, research, and provision of comprehensive care to the nation's population.

The ADFM Board of Directors has taken a strong stance that we need to address diversity and inclusion in an authentic and deeper way if we are to be true to our values and mission of promoting health and health equity. In assessing this newest priority, the ADFM Board of Directors recognizes that as chairs and senior administrators we are, by definition, leaders in advancing these values and this priority in the academy. It is incumbent that we offer our membership the means to examine our departments and, when possible, our institutions with respect to diversity, inclusion, and health equity. Furthermore, we recognize the need to share these concepts and how we operationally apply them with the entire Family of Family Medicine and medicine in general.

A new ADFM Diversity, Inclusion, and Health Equity (DIHE) Task force has been formed to carry out these functions. Among specific tasks identified by the Task force are:

- To promote a shared understanding among ADFM members of concepts and terms. Drawing on established work, we will provide definitions to clarify the 3 concepts (Diversity, Inclusion, and Health Equity) of this initiative.

- To incorporate DIHE concepts into the work of all ADFM committees and governance, holding ourselves accountable in all aspects of our work as an organization. We will accomplish these tasks in a manner that includes transparency and adherence to our guiding principles.

- To develop a template of implementation plans for use by individual departments as appropriate and consistent with their home institution plans.

- To advance these concepts in our interorganizational work, such as through the Council of
Academic Family Medicine (CAFM) and the American Academy of Family Physician's Center for Diversity and Health Equity (CDHE) (external)

The Task force alone cannot do the work. This is a CALL TO ACTION! We need every Chair and Administrator in our member Departments to own this work with us. We will:

- Educate

-What is the connection between the 3 concepts - Diversity, Inclusion, and Health Equity? How they are related, yet different?

- Connect these concepts to higher organizational performance and to achieving better health and health equity outcomes for patients and communities

- Inspire and Develop a vision

- Consider methodologies which bring these concepts/themes into the heart of ADFM and the broader Family of Family Medicine

- Develop a framework which helps an institution conduct a gap-analysis with consideration of solutions to improve Diversity, Inclusion, and Equity. Potential modalities for dissemination are:

- Webinars: Similar to our highly popular "joy in practice" webinar series, conduct webinars to educate chairs and administrators about this framework

- 1:1 Consultations

- Identify and disseminate best practices for implementation of strategies to address gaps

We have reviewed working definitions for diversity, inclusion, and health equity as we set out to do the "what and how" of our work. These definitions, drawn from AAMC Group on Diversity and Inclusion (https://www.aamc.org/members/gdi/), Healthy People 2020 (jhttp://www.healthypeople.gov), and AAFP (https://www.aafp.org/about/policies/all/healthequity.html), offer a compendium of others' efforts and a starting point in our own assessment. These will be included on our website. However, the unique nature of our work-being responsible for teaching, research, and clinical care-requires further inquiry into how we will operationalize these definitions and thus we need input from the membership. The end result will be a product that is useful to the membership and its various constituencies.

We invite all members of the Family of Family Medicine to join us in this endeavor. We will only achieve our goals with the maximal participation of all of you. And we can only fulfill our roles as leaders when we achieve these goals.

Task force Co-Authors: Mark Jobuson (Chair), Monty Douglas, Kevin Grumbach, Myra Muramoto, Anna Ramanathan, Beth Wilson, Pbilip Zazove, Ardis Davis (Staff) 